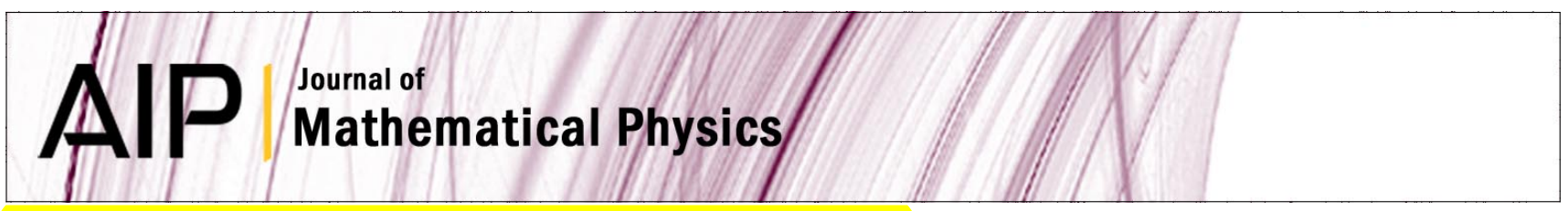

\title{
Quantum-to-classical rate distortion coding
}

Nilanjana Datta, Min-Hsiu Hsieh, Mark M. Wilde, and Andreas Winter

Citation: J. Math. Phys. 54, 042201 (2013); doi: 10.1063/1.4798396

View online: http://dx.doi.org/10.1063/1.4798396

View Table of Contents: http://jmp.aip.org/resource/1/JMAPAQ/v54/i4

Published by the American Institute of Physics.

\section{Additional information on J. Math. Phys.}

Journal Homepage: http://jmp.aip.org/

Journal Information: http://jmp.aip.org/about/about_the_journal

Top downloads: http://jmp.aip.org/features/most_downloaded

Information for Authors: http://jmp.aip.org/authors

\section{ADVERTISEMENT}

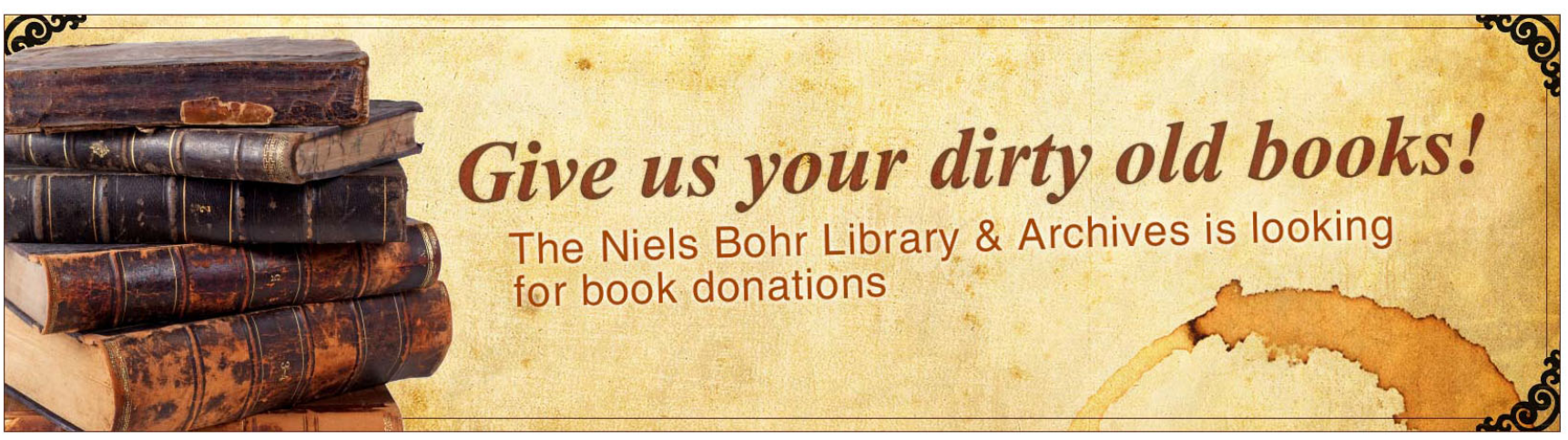




\title{
Quantum-to-classical rate distortion coding
}

\author{
Nilanjana Datta, ${ }^{1}$ Min-Hsiu Hsieh, ${ }^{2}$ Mark M. Wilde, ${ }^{3}$ and Andreas Winter ${ }^{4}$ \\ ${ }^{1}$ Statistical Laboratory, University of Cambridge, Wilberforce Road, Cambridge CB3 OWB, \\ United Kingdom \\ ${ }^{2}$ Centre for Quantum Computation \& Intelligent Systems, Faculty of Engineering and \\ Information Technology, University of Technology, Sydney, P.O. Box 123, Broadway NSW \\ 2007, Australia \\ ${ }^{3}$ School of Computer Science, McGill University, Montreal, Quebec H3A 2A7, Canada \\ ${ }^{4}$ ICREA - Institució Catalana de Recerca i Estudis Avançats, Pg. Lluis Companys 23, \\ ES-08010 Barcelona, Spain; Física Teòrica: Informació i Fenomens Quàntics, Universitat \\ Autònoma de Barcelona, ES-08193 Bellaterra (Barcelona), Spain; Department of \\ Mathematics, University of Bristol, Bristol BS8 1TW, United Kingdom; and Centre for \\ Quantum Technologies, National University of Singapore, Singapore
}

(Received 19 January 2013; accepted 11 March 2013; published online 2 April 2013)

\begin{abstract}
We establish a theory of quantum-to-classical rate distortion coding. In this setting, a sender Alice has many copies of a quantum information source. Her goal is to transmit a classical description of the source, obtained by performing a measurement on it, to a receiver Bob, up to some specified level of distortion. We derive a single-letter formula for the minimum rate of classical communication needed for this task. We also evaluate this rate in the case in which Bob has some quantum side information about the source. Our results imply that, in general, Alice's best strategy is a non-classical one, in which she performs a collective measurement on successive outputs of the source. () 2013 American Institute of Physics. [http://dx.doi.org/10.1063/1.4798396]
\end{abstract}

\section{INTRODUCTION}

A fundamental task in quantum information theory is the reliable compression of information emitted by a quantum information source, to enable efficient storage of the data. Schumacher ${ }^{15}$ proved that, for a memoryless source, the optimal rate of lossless data compression (in which the original data are recovered perfectly in the limit of asymptotically many copies of the source) is given by the von Neumann entropy of the source. The corresponding rate for a classical source is given by its Shannon entropy. ${ }^{18}$

In realistic applications it may be possible, however, to tolerate imperfect recovery of the signals, and hence allow for a bounded distortion of the original information. In fact, this may even be necessitated by the lack of sufficient storage. These considerations have led to the development of rate distortion theory, ${ }^{3}$ which is the theory of lossy data compression. The fundamental results of classical rate distortion theory are attributed to Shannon ${ }^{19}$ and date back to 1948. Its quantum counterpart was introduced by Barnum ${ }^{2}$ and developed further in Refs. 5 and 9. Recently, Datta et al. identified a regularized expression for the quantum rate distortion function as well as a single-letter expression for the entanglement-assisted quantum rate distortion function. ${ }^{8}$

In this paper, we consider the situation in which a party (say, Alice) obtains many copies of a quantum information source described by a quantum state, and she already has a description of the source in terms of its density operator. She is only allowed to perform measurements on the source. Her aim is to suitably compress the classical data resulting from her measurements and send it to another party (say, Bob) such that, upon decompression, the data recovered by Bob have a fixed level of distortion from the quantum source (specified by a suitable distortion observable). Alice is allowed to perform any measurement that she wishes on the source states to produce a classical sequence, with the requirement that the average symbolwise distortion of this sequence be no larger than some prescribed amount. Analogous to previous terminology 
used in quantum information theory, we refer to this as quantum-to-classical rate distortion theory, since it deals with an analysis of the trade-off between the optimal rate of compression of the data obtained by measurements on the quantum source, and the allowed distortion on the recovered classical data. This trade-off is quantified by the quantum-to-classical rate-distortion function.

Another way of emphasizing the relevance of quantum-to-classical rate distortion theory is by adopting the perspective that all classical data arise from a measurement of a quantum state. This is especially important in cases where the source is truly non-classical, such as an atomic decaying process or a highly attenuated laser. ${ }^{27}$ In particular, we can imagine that a memoryless classical source arises from an appropriate measurement on the states emitted by a quantum source, and the resulting classical data have some description or characterization of the original quantum source. Thus, this perspective necessitates a revision of Shannon's rate-distortion theory ${ }^{19}$ by allowing for an arbitrary measurement to be performed on the original quantum source. A naive approach to this setting would be to measure each individual output of the quantum source, treat the resulting classical data as information emitted by a classical source, and then apply Shannon's rate-distortion theory to the latter.

Here, we instead allow for collective measurements on the outputs of the source, and our approach is to apply a derandomized measurement compression protocol to achieve this task. ${ }^{24} \mathrm{We}$ find a single-letter formula for the quantum-to-classical rate distortion function, expressed as a minimization of the quantum mutual information over all maps that meet the distortion constraint. Our result implies that, in general, a quantum strategy is needed to achieve optimal compression rates and that Shannon's rate-distortion theory is insufficient in this setting. This result is analogous to the fact that collective measurements are needed in general in the well-known Holevo-SchumacherWestmoreland theorem ${ }^{12,17}$ regarding classical communication over quantum channels (see Ref. 11 for an explicit example of a channel for which collective measurements outperform classical strategies).

In the classical setting, the optimal rate of data compression can be reduced if the decoder (Bob) has some side information at his disposal. The first discovery in this direction is due to Slepian and Wolf, ${ }^{20}$ who showed that the optimal lossless compression rate is given by the entropy of the source conditioned on the side information. Wyner and Ziv extended these results to the case of lossy classical data compression with classical side information. ${ }^{26}$ For the quantum setting, one might imagine that quantum side information is available at the decoder. In Ref. 10, Devetak and Winter proved that if Bob has quantum side information at his disposal, then the optimal lossless compression rate for a classical information source is reduced from the Shannon entropy of the source by the Holevo information between the source and the quantum side information. The case of lossy classical data compression with quantum side information, which is a quantum generalization of the Wyner-Ziv problem, was studied by Luo and Devetak. ${ }^{13}$

We also study the effect of quantum side information on the above-mentioned quantum-toclassical rate distortion function. In particular, we consider the case in which some quantum side information about the original quantum source is available to Bob. He is allowed to use this information to recover the classical data obtained from Alice's measurements on the source states. We also let Alice and Bob share common randomness. In this case, we find a single-letter formula for the corresponding quantum-to-classical rate distortion function. One of our assumptions in this setting is that the process of compression and decompression only causes a negligible disturbance to the quantum side information. This assumption can be justified by the possibility of Bob wanting to use the quantum side information in some future protocol. Our result improves upon the aforementioned work of Luo and Devetak ${ }^{13}$ in the sense that we find a matching single-letter converse for this setting. The achievability part of the proof of this theorem exploits measurement compression with quantum side information. ${ }^{22}$

The paper is organized as follows. We summarize some necessary definitions and prerequisites in Sec. II and in Sec. III, we review the concept of a distortion observable (originally introduced in Refs. 5 and 25). In Sec. IV, we introduce the task of quantum-to-classical rate distortion coding, define a suitable distortion observable, and derive an expression for the quantum-to-classical rate distortion function. In Sec. V, we study quantum-to-classical rate distortion in the presence of 
quantum side information and common randomness. The main results of this paper are given by Theorem 3 of Sec. IV and Theorems 5 and 6 of Sec. V.

\section{NOTATIONS AND DEFINITIONS}

Let $\mathcal{B}(\mathcal{H})$ denote the algebra of linear operators acting on a finite-dimensional Hilbert space $\mathcal{H}$ and let $\mathcal{D}(\mathcal{H})$ denote the set of positive operators of unit trace (states) acting on $\mathcal{H}$. For any given pure state $|\psi\rangle \in \mathcal{H}$ we denote the projector $|\psi\rangle\langle\psi|$ simply as $\psi$. The trace distance between two operators $A$ and $B$ is given by $\|A-B\|_{1} \equiv \operatorname{Tr}|A-B|$, where $|C| \equiv \sqrt{C^{\dagger} C}$. Throughout this paper we restrict our considerations to finite-dimensional Hilbert spaces, and we take the logarithm to base 2 . In the following we denote a completely positive trace-preserving (CPTP) map $\mathcal{N}: \mathcal{B}\left(\mathcal{H}_{A}\right) \rightarrow \mathcal{B}\left(\mathcal{H}_{B}\right)$ simply as $\mathcal{N}^{A \rightarrow B}$. Similarly we denote an isometry $U: \mathcal{B}\left(\mathcal{H}_{A}\right) \rightarrow \mathcal{B}\left(\mathcal{H}_{B} \otimes \mathcal{H}_{E}\right)$ simply as $U^{A} \rightarrow B E$. The identity map on states in $\mathcal{D}\left(\mathcal{H}_{A}\right)$ is denoted as $\mathrm{id}_{A}$.

The von Neumann entropy of a state $\rho \in \mathcal{D}\left(\mathcal{H}_{A}\right)$ is defined as $H(\rho) \equiv-\operatorname{Tr}\{\rho \log \rho\}$. In the following we use $H(A \mid B)_{\rho}$ and $I(A ; B)_{\rho}$ to respectively denote the conditional quantum entropy and the quantum mutual information of a bipartite state $\rho_{A B}$, and $I(A ; C \mid B)_{\sigma}$ to denote the conditional quantum mutual information for a tripartite state $\sigma_{A B C}$ (see, e.g., Refs. 14 and 21). We also employ the following properties of the quantum mutual information:

Lemma 1 (Quantum data processing inequality $\left.{ }^{16,21}\right)$. If $\omega_{A B^{\prime}}=\left(\mathrm{id}_{A} \otimes \mathcal{N}^{B \rightarrow B^{\prime}}\right) \sigma_{A B}$, where $\mathcal{N}^{B \rightarrow B^{\prime}}$ is a CPTP map, then

$$
I(A ; B)_{\sigma} \geq I\left(A ; B^{\prime}\right)_{\omega}
$$

Lemma 2 (Superadditivity of the quantum mutual information ${ }^{8}$ ). The mutual information is superadditive in the sense that, for any $C P T P$ map $\mathcal{N}^{A_{1} A_{2} \rightarrow B_{1} B_{2}}$,

$$
I\left(R_{1} R_{2} ; B_{1} B_{2}\right)_{\sigma} \geq I\left(R_{1} ; B_{1}\right)_{\sigma}+I\left(R_{2} ; B_{2}\right)_{\sigma},
$$

where

$$
\sigma_{R_{1} R_{2} B_{1} B_{2}}=\mathcal{N}^{A_{1} A_{2} \rightarrow B_{1} B_{2}}\left(\phi_{R_{1} A_{1}} \otimes \varphi_{R_{2} A_{2}}\right)
$$

and $\phi_{R_{1} A_{1}}$ and $\varphi_{R_{2} A_{2}}$ are pure bipartite states.

In proving our first theorem (Theorem 3 of Sec. IV) we make use of the "measurement compression" theorem (Theorem 2 of Ref. 24). The latter specifies an optimal two-dimensional rate region characterizing the resources (namely, common randomness and classical communication) needed for an asymptotically faithful simulation of a measurement on a quantum state. For an exact statement of the theorem, see Refs. 22 and 24. Here we give a brief description of its content. Let $\psi_{R A}^{\rho}$ denote the purification of a quantum state $\rho \in \mathcal{D}\left(\mathcal{H}_{A}\right)$, multiple copies of which are in Alice's possession. Suppose Alice does a measurement, given by a positive operator-valued measure (POVM) $\Lambda$ $\equiv\left\{\Lambda_{x}\right\}$, on each of the systems in her possession. In the ideal measurement compression protocol, the state of the classical registers containing Alice's measurement outcomes and the purifying reference systems $R$ is equivalent to many copies of the following state:

$$
\sigma_{X R} \equiv \sum_{x}|x\rangle\left\langle\left. x\right|_{X} \otimes \operatorname{Tr}_{A}\left\{\left(I_{R} \otimes \Lambda_{x}\right) \psi_{R A}^{\rho}\right\} .\right.
$$

The measurement compression theorem asserts that if Alice and Bob share $n H(X \mid R)_{\sigma}$ bits of common randomness, then it is possible for them to simulate the measurement $\Lambda^{\otimes n}$ on the state $\rho^{\otimes n}$ with approximately $n I(X ; R)_{\sigma}$ bits of classical communication, for $n$ large enough. The simulation becomes faithful in the limit $n \rightarrow \infty$, in the sense that a verifying party who possesses the classical registers and the reference systems cannot distinguish between the output of the simulation and the ideal protocol. If no common randomness is present and Alice is required to obtain the outcomes of the measurement in addition to Bob, then the classical communication needed is equal to the Shannon entropy $H(X)_{\sigma}$. In the above, $H(X \mid R)_{\sigma}$ and $I(X ; R)_{\sigma}$ respectively denote the conditional 
entropy and the mutual information of the state $\sigma_{X R}$ defined above. For a more detailed statement of the theorem, see the proof of Theorem 3 in Sec. IV.

\section{DISTORTION OBSERVABLES}

As discussed in the Introduction, in rate distortion theory one allows the data which are recovered after the compression-decompression scheme to be distorted by some finite amount from the original data. There are various possible choices of the distortion measure, depending on the nature of the application. For example, in classical rate distortion theory, the Hamming distance and the mean squared error are natural choices of the distortion measure. ${ }^{3,6}$ In quantum rate distortion theory, the distortion measure is usually defined in terms of the entanglement fidelity (see, e.g., Refs. 2 and 8 and references therein). However, since the distortion is a physical quantity, it is natural to associate with it an observable in the quantum setting (as discussed in Sec. II of Ref. 5 and in unpublished work $^{25}$ ). This is reviewed below.

In the classical setting, let $x \in \mathcal{X}$ denote the letters of a source alphabet and let $y \in \mathcal{Y}$ denote the letters of a reconstruction alphabet. Then to determine the distortion between an input and output letter, one defines a non-negative cost function $d(x, y)$ (e.g., the Hamming distance or the squared error), and the average distortion is then given by

$$
\sum_{x} \sum_{y} p(x) q(y \mid x) d(x, y),
$$

where $q(y \mid x)$ is the conditional probability of getting the letter $y$ after reconstruction when the source letter is $x$ and $p(x)$ is the probability of source letter $x$.

In the quantum case, one defines a distortion observable $\Delta .^{5}$ For example, suppose that $\Delta$ is given by

$$
\Delta=\sum_{x} \sum_{y} d(x, y)|x\rangle\langle x|\otimes| y\rangle\langle y|,
$$

where $|x\rangle$ are the Schmidt vectors of the following purification of the source state $\rho$ :

$$
\left|\psi_{R A}^{\rho}\right\rangle=\sum_{x} \sqrt{\lambda_{x}}|x\rangle_{R}|x\rangle_{A}
$$

so that $\rho=\operatorname{Tr}_{R}\left\{\psi_{R A}^{\rho}\right\}$.

Then we recover the expression (3) for the average distortion in the classical case as follows. Let $\Phi: \mathcal{B}\left(\mathcal{H}_{A}\right) \mapsto \mathcal{B}\left(\mathcal{H}_{B}\right)$ denote a map on the source state. Then the average distortion is given by

$$
\begin{aligned}
& \operatorname{Tr}\left\{\Delta\left((\operatorname{id} \otimes \Phi)\left(\psi_{R A}^{\rho}\right)\right)\right\} \\
& =\operatorname{Tr}\left\{\left(\sum _ { x , y } d ( x , y ) | x \rangle \langle x | _ { R } \otimes | y \rangle \langle y | _ { B } ) \left(\sum_{x^{\prime}, y^{\prime}} \sqrt{\lambda_{x^{\prime}}} \sqrt{\lambda_{y^{\prime}}}\left|x^{\prime}\right\rangle\left\langle\left. y^{\prime}\right|_{R} \otimes \Phi\left(\left|x^{\prime}\right\rangle\left\langle\left. y^{\prime}\right|_{A}\right)\right)\right\}\right.\right.\right. \\
& =\sum_{x, y} d(x, y) \lambda_{x}\left\langley \left|\Phi \left(|x\rangle\left\langle\left. x\right|_{A}\right)|y\rangle .\right.\right.\right.
\end{aligned}
$$

Let us define $q(y \mid x) \equiv\left\langle y\left|\Phi\left(|x\rangle\left\langle\left. x\right|_{A}\right)|y\rangle\right.\right.\right.$ since it can be interpreted as the conditional probability of the map $\Phi$ yielding the letter $y$, given that the source letter was $x$. Then setting $p(x)=\lambda_{x}$, (since $\lambda_{x}$, being an eigenvalue of $\rho$, is a probability), we recover the expression for the classical average distortion as in (3).

\section{QUANTUM-TO-CLASSICAL RATE-DISTORTION CODING}

Consider a memoryless quantum information source $\left\{\rho, \mathcal{H}_{A}\right\}$. In quantum-to-classical (q-c) rate distortion, Alice starts with $n$ copies $\rho^{\otimes n}$ of the source state and performs a POVM $\Lambda^{(n)}=\left\{\Lambda_{x^{n}}\right\}$ on it, with the POVM elements $\Lambda_{x^{n}} \in \mathcal{B}\left(\mathcal{H}_{A}^{\otimes n}\right)$ being indexed by classical sequences $x^{n} \in \mathcal{X}^{n}(\mathcal{X}$ being a finite alphabet), which correspond to the different possible outcomes of the measurement. It 


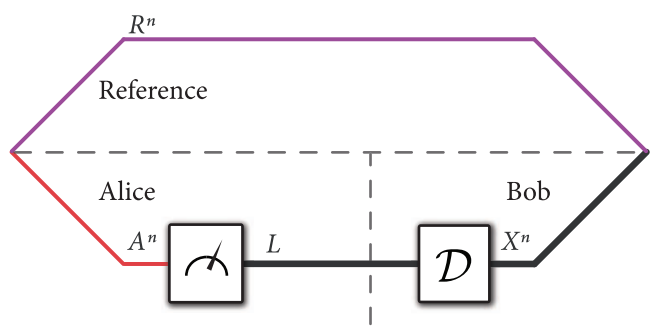

FIG. 1. The most general protocol for quantum-to-classical rate-distortion coding. Alice has many copies of the quantum information source, on which she performs a collective measurement with classical output $L$. She sends the variable $L$ over noiseless classical bit channels to Bob. Bob then performs a classical decoding map on $L$ that outputs the classical sequence $X^{n}$. The average deviation of this sequence from the quantum source, according to some distortion observable, provides a measure of the distortion caused by this protocol.

is convenient to define a measurement map $\mathcal{M}_{\Lambda^{(n)}}$ corresponding to the POVM $\Lambda^{(n)}$ as follows: For any $\sigma_{n} \in \mathcal{D}\left(\mathcal{H}_{A}^{\otimes n}\right)$,

$$
\mathcal{M}_{\Lambda^{(n)}}\left(\sigma_{n}\right) \equiv \sum_{x^{n} \in \mathcal{X}^{n}} \operatorname{Tr}\left\{\Lambda_{x^{n}} \sigma_{n}\right\}\left|x^{n}\right\rangle\left\langle x^{n}\right| .
$$

The above specifies that with probability $\operatorname{Tr}\left\{\Lambda_{x^{n}} \sigma_{n}\right\}$ the outcome of the POVM $\Lambda^{(n)}$ on the state $\sigma_{n}$ is given by the classical sequence $x^{n}$. Figure 1 depicts the most general protocol for quantum-toclassical rate-distortion coding.

If $\psi_{R^{n} A^{n}}^{\rho}$ denotes a purification of $\rho^{\otimes n}$, then the following bipartite state characterizes both the classical outcome of the POVM $\Lambda^{(n)}$ on $\rho^{\otimes n}$ and the post-measurement state of the purifying reference system:

$$
\begin{aligned}
\sigma_{R^{n} X^{n}} & \equiv\left(\operatorname{id}_{R^{n}} \otimes \mathcal{M}_{\Lambda^{(n)}}\right)\left(\psi_{R^{n} A^{n}}^{\rho}\right) \\
& =\sum_{x^{n}} \operatorname{Tr}_{A^{n}}\left\{\left(I_{R^{n}} \otimes \Lambda_{x^{n}}\right) \psi_{R^{n} A^{n}}^{\rho}\right\} \otimes\left|x^{n}\right\rangle\left\langle\left. x^{n}\right|_{X^{n}} .\right.
\end{aligned}
$$

We define the q-c distortion measure for a state $\rho \in \mathcal{D}\left(\mathcal{H}_{A}\right)$ with purification $\left|\psi_{R A}^{\rho}\right\rangle$ and a POVM $\Lambda=\left\{\Lambda_{x}\right\}$ as

$$
d\left(\rho, \mathcal{M}_{\Lambda}\right) \equiv \operatorname{Tr}\left(\Delta\left(\mathrm{id} \otimes \mathcal{M}_{\Lambda}\right)\left(\psi_{R A}^{\rho}\right)\right),
$$

where $\mathcal{M}_{\Lambda}$ is the measurement map corresponding to $\Lambda$, and $\Delta$ is a q-c distortion observable given by

$$
\Delta \equiv \Delta_{R X} \equiv \sum_{x} \Delta_{x} \otimes|x\rangle\langle x|
$$

with $\Delta_{x} \geq 0$.

A q-c rate distortion code of rate $R$ is given by a POVM $\Lambda^{(n)}$ with $\left\lfloor 2^{n R}\right\rfloor$ outcomes, i.e., $\Lambda^{(n)}=\left\{\Lambda_{x^{n}}\right\}$ with

$$
\#\left\{x^{n} \in \mathcal{X}^{n}: \Lambda_{x^{n}} \neq 0\right\}=\left\lfloor 2^{n R}\right\rfloor .
$$

To define the average distortion resulting from this POVM, we consider a symbolwise q-c distortion observable

$$
\Delta^{(n)} \equiv \frac{1}{n} \sum_{i=1}^{n} \Delta_{R_{i} X_{i}} \otimes I_{R X}^{\otimes[n] \backslash i}
$$

where each operator $\Delta_{R_{i} X_{i}}$ is of the form (10) and $I_{R X}^{\otimes[n] \backslash i}$ denotes the identity operator acting on all but the $i$ th member of the tensor-product of Hilbert spaces $\left(\mathcal{H}_{R} \otimes \mathcal{H}_{X}\right)^{\otimes n}$. The average distortion is 
then defined as

$$
\begin{aligned}
\bar{d}\left(\rho, \mathcal{M}_{\Lambda^{(n)}}\right) & \equiv \operatorname{Tr}\left(\Delta^{(n)}\left(\operatorname{id}_{R^{n}} \otimes \mathcal{M}_{\Lambda^{(n)}}\right) \psi_{R^{n} A^{n}}^{\rho}\right) \\
& =\frac{1}{n} \sum_{i=1}^{n} \operatorname{Tr}\left(\Delta_{R_{i} X_{i}} \sigma_{R_{i} X_{i}}\right),
\end{aligned}
$$

where $\sigma_{R_{i} X_{i}}=\operatorname{Tr}_{\neq i} \sigma_{R^{n} X^{n}}$, with $\sigma_{R^{n} X^{n}} \equiv\left(\operatorname{id}_{R^{n}} \otimes \mathcal{M}_{\Lambda^{(n)}}\right) \psi_{R^{n} A^{n}}^{\rho}$.

For any $R, D \geq 0$, the pair $(R, D)$ is said to be an achievable q-c rate distortion pair if there exists a sequence of POVMs $\left\{\Lambda^{(n)}\right\}_{n \geq 1}$ of rate $R$ such that

$$
\lim _{n \rightarrow \infty} \bar{d}\left(\rho, \mathcal{M}_{\Lambda^{(n)}}\right) \leq D
$$

The q-c rate distortion function is then defined as

$$
R^{q c}(D) \equiv \inf \{R:(R, D) \text { achievable }\} .
$$

The following theorem provides a single-letter expression for $R^{q c}(D)$.

Theorem 3. For a memoryless quantum information source $\left\{\rho, \mathcal{H}_{A}\right\}$, a quantum-to-classical distortion observable $\Delta_{R X}$, and any given distortion $D \geq 0$, the quantum-to-classical rate distortion function is given by

$$
R^{q c}(D)=\min _{\substack{\operatorname{PovM} \Lambda=\left\{\Lambda_{x}\right\} \\ d\left(\rho, \mathcal{M}_{\Lambda}\right) \leq D}} I(X ; R)_{\sigma}
$$

where $d\left(\rho, \mathcal{M}_{\Lambda}\right)$ is defined through (9) and (10) and

$$
\sigma_{R X} \equiv\left(\operatorname{id}_{R} \otimes \mathcal{M}_{\Lambda}\right)\left(\psi_{R A}^{\rho}\right)=\sum_{x} \operatorname{Tr}_{A}\left\{\left(\operatorname{id}_{R} \otimes \Lambda_{x}\right) \psi_{R A}^{\rho}\right\} \otimes|x\rangle\left\langle\left. x\right|_{X} .\right.
$$

Proof. We first give the proof of achievability, which follows directly from the measurement compression theorem $^{24}$ (summarized briefly in Sec. II). Our approach is similar to one used before: ${ }^{23}$ exploit a channel simulation protocol and derandomize the common randomness consumed by this protocol. So, fix the POVM $\Lambda=\left\{\Lambda_{x}\right\}$ that minimizes the RHS of (15). Thus we have

$$
d\left(\rho, \mathcal{M}_{\Lambda}\right)=\operatorname{Tr}\left[\Delta\left(\mathrm{id}_{R} \otimes \mathcal{M}_{\Lambda}\right)\left(\psi_{R A}^{\rho}\right)\right] \leq D
$$

In Ref. 24, it was proved that there exists a finite set of POVMs $\left\{\Lambda^{(m)}=\left\{\Lambda_{x^{n}}^{(m)}\right\}_{x^{n} \in \mathcal{X}^{n}}: m\right.$ $=1,2, \ldots, M\}$, each having at most $L$ outcomes, i.e., $\#\left\{x^{n} \in \mathcal{X}^{n}: \Lambda_{x^{n}}^{(m)} \neq 0\right\} \leq L$, with

$$
L=2^{n I(R ; X)_{\sigma}+O(\sqrt{n})},
$$

such that for any $\varepsilon>0$ and $n$ large enough, the POVM $\tilde{\Lambda}^{(n)}=\left\{\tilde{\Lambda}_{x^{n}}\right\}$ defined as

$$
\tilde{\Lambda}^{(n)} \equiv \frac{1}{M} \sum_{m=1}^{M} \Lambda^{(m)}
$$

satisfies the following condition:

$$
\left\|\left(\operatorname{id}_{R^{n}} \otimes \mathcal{M}_{\tilde{\Lambda}^{(n)}}\right) \psi_{R^{n} A^{n}}^{\rho}-\left(\operatorname{id}_{R^{n}} \otimes \mathcal{M}_{\left.\Lambda^{\otimes n}\right)}\right) \psi_{R^{n} A^{n}}^{\rho}\right\|_{1} \leq \varepsilon
$$

where for any sequence $x^{n}=x_{1} \ldots x_{n} \in \mathcal{X}^{n}$ we have $\Lambda_{x^{n}}=\Lambda_{x_{1}} \otimes \ldots \Lambda_{x_{n}}$. Further, due to our choice (12) of a symbolwise q-c distortion observable $\Delta^{(n)}$, we have that

$$
\bar{d}\left(\rho, \mathcal{M}_{\Lambda^{\otimes n}}\right)=\operatorname{Tr}\left[\Delta^{(n)}\left(\operatorname{id}_{R^{n}} \otimes \mathcal{M}_{\Lambda^{\otimes n}}\right) \psi_{R^{n} A^{n}}^{\rho}\right]=d\left(\rho, \mathcal{M}_{\Lambda}\right) .
$$

From (20), we know that the protocol for simulating the tensor-product measurement has measurement encodings $\left\{\Lambda_{l}^{(m)}\right\}$. Let $\mathcal{D}^{(m)}(l)$ denote the corresponding classical decodings which construct the sequences $x^{n}$ from the values of $l$ and $m$, where $l$ is the measurement outcome and $m$ is the common randomness. Then

$$
\|\left(\operatorname{id}_{R^{n}} \otimes \mathcal{M}_{\Lambda^{\otimes n}}\right)\left(\psi_{R^{n} A^{n}}^{\rho}\right)-\frac{1}{M} \sum_{m, l} \operatorname{Tr}_{A^{n}}\left\{\left(\operatorname{id}_{R^{n}} \otimes \Lambda_{l}^{(m)}\right) \psi_{R^{n} A^{n}}^{\rho}\right\} \otimes\left|\mathcal{D}^{(m)}(l)\right\rangle\left\langle\mathcal{D}^{(m)}(l)\right| \|_{1} \leq \varepsilon .
$$


Then using (17) we obtain a bound on the average distortion resulting from the action of the POVM $\tilde{\Lambda}^{(n)}$ on the source state $\rho^{\otimes n}$ as follows:

$$
\begin{aligned}
& \bar{d}\left(\rho, M_{\tilde{\Lambda}^{(n)}}\right) \\
& =\operatorname{Tr}\left\{\Delta^{(n)} \frac{1}{|M|} \sum_{m, l} \operatorname{Tr}_{A^{n}}\left\{\left(\operatorname{id}_{R^{n}} \otimes \Lambda_{l}^{(m)}\right) \psi_{R^{n}}^{\rho} A^{n}\right\} \otimes\left|\mathcal{D}^{(m)}(l)\right\rangle\left\langle\mathcal{D}^{(m)}(l)\right|\right\} \\
& =\frac{1}{|M|} \sum_{m} \operatorname{Tr}\left\{\Delta^{(n)} \sum_{l} \operatorname{Tr}_{A^{n}}\left\{\left(\operatorname{id}_{R^{n}} \otimes \Lambda_{l}^{(m)}\right) \psi_{R^{n} A^{n}}^{\rho}\right\} \otimes\left|\mathcal{D}^{(m)}(l)\right\rangle\left\langle\mathcal{D}^{(m)}(l)\right|\right\} \\
& =\frac{1}{|\mathcal{M}|} \sum_{m} d_{\max } \operatorname{Tr}\left\{\frac{\Delta^{(n)}}{d_{\max }} \sum_{l} \operatorname{Tr}_{A^{n}}\left\{\left(\operatorname{id}_{R^{n}} \otimes \Lambda_{l}^{(m)}\right) \psi_{R^{n} A^{n}}^{\rho}\right\} \otimes\left|\mathcal{D}^{(m)}(l)\right\rangle\left\langle\mathcal{D}^{(m)}(l)\right|\right\} \\
& \leq d_{\max } \operatorname{Tr}\left\{\frac{\Delta^{(n)}}{d_{\max }}\left(\operatorname{id}_{R^{n}} \otimes \mathcal{M}_{\Lambda^{\otimes n}}\right)\left(\psi_{R^{n} A^{n}}^{\rho}\right)\right\}+d_{\max } \varepsilon \\
& \leq D+d_{\max } \varepsilon,
\end{aligned}
$$

where $d_{\max }$ is the maximum eigenvalue of $\Delta^{(n)}$. Also, in the above, we see how it is possible to derandomize the common randomness: there exists a choice of the $m$ such that

$$
\operatorname{Tr}\left\{\Delta^{(n)} \sum_{l} \operatorname{Tr}_{A^{n}}\left\{\operatorname{id}_{R^{n}} \otimes \Lambda_{l}^{(m)} \psi_{R^{n} A^{n}}^{\rho}\right\} \otimes\left|\mathcal{D}^{(m)}(l)\right\rangle\left\langle\mathcal{D}^{(m)}(l)\right|\right\} \leq D+d_{\max } \varepsilon
$$

Hence,

$$
\lim _{n \rightarrow \infty} \bar{d}\left(\rho, \mathcal{D}_{n} \circ \mathcal{M}_{\Lambda^{(n)}}\right) \leq D
$$

Thus, a measurement compression protocol directly yields a q-c rate distortion protocol.

Now we give a proof for the converse. Let $\Lambda^{(n)}: A^{n} \mapsto L$ be a POVM with $\Lambda^{(n)}=\left\{\Lambda_{l}^{(n)}\right\}$, and let $\mathcal{D}_{n}: L \mapsto X^{n}$ be a decoding map (with $L$ and $X^{n}$ denoting classical systems) such that

$$
\lim _{n \rightarrow \infty} \bar{d}\left(\rho, \mathcal{D}_{n} \circ \mathcal{M}_{\Lambda^{(n)}}\right) \leq D
$$

where $\mathcal{M}_{\Lambda^{(n)}}$ is the measurement map corresponding to the POVM $\Lambda^{(n)}$. Defining $\sigma_{R^{n} L} \equiv$ $\left(\operatorname{id}_{R^{n}} \otimes \mathcal{M}_{\Lambda^{(n)}}\right) \psi_{R^{n} A^{n}}^{\rho}$, we have $\sigma_{L}=\sum_{l} \operatorname{Tr}\left(\Lambda_{l}^{(n)} \rho^{\otimes n}\right)|l\rangle\langle l|$ and

$$
\begin{aligned}
n R & \geq H(L)_{\sigma} \\
& \geq I\left(L ; R^{n}\right)_{\sigma} \\
& \geq I\left(X^{n} ; R^{n}\right)_{\omega} .
\end{aligned}
$$

The first inequality holds because the entropy $H(L)_{\sigma}$ is upper bounded by the entropy $n R$ of the uniform distribution. In the second line, the inequality follows because $I\left(L ; R^{n}\right)_{\sigma}=H(L)_{\sigma}$ - $H\left(L \mid R^{n}\right)_{\sigma}$ and $H\left(L \mid R^{n}\right)_{\sigma} \geq 0$ since $L$ is classical. In the third line, $\omega_{X^{n} R^{n} D} \equiv\left(\operatorname{id}_{R^{n}} \otimes \mathcal{D}_{n}\right) \sigma_{R^{n} L}$. This inequality follows from the quantum data processing inequality (Lemma 1). Continuing, we 
have

$$
\begin{aligned}
\text { RHS of (24) } & \geq \sum_{i=1}^{n} I\left(X_{i} ; R_{i}\right) \\
& \geq \sum_{i=1}^{n} R^{q c}\left(d\left(\rho, \mathcal{F}_{n}^{(i)}\right)\right) \\
& =n \sum_{i=1}^{n} \frac{1}{n} R^{q c}\left(d\left(\rho, \mathcal{F}_{n}^{(i)}\right)\right) \\
& \geq n R^{q c}\left(\sum_{i=1}^{n} \frac{1}{n} d\left(\rho, \mathcal{F}_{n}^{(i)}\right)\right) \\
& \geq n R^{q c}(D),
\end{aligned}
$$

for $n$ sufficiently large. In the above, $\mathcal{F}_{n}^{(i)}$ is the marginal operation on the $i$ th copy of the source space induced by the overall operation $\mathcal{D}_{n} \circ \mathcal{M}_{\Lambda^{(n)}}$. The first inequality follows from the superadditivity of the quantum mutual information (Lemma 2). The second inequality follows from the fact that the map $\mathcal{F}_{n}^{(i)}$ has distortion $d\left(\rho, \mathcal{F}_{n}^{(i)}\right)$, which, by definition (14), is lower bounded by the q-c rate distortion function corresponding to this distortion. The last two inequalities follow from the convexity of the q-c rate distortion function, from the assumption that the average distortion of the protocol is less than or equal to $D$ for $n$ large enough, i.e.,

$$
\sum_{i=1}^{n} \frac{1}{n} d\left(\rho, \mathcal{F}_{n}^{(i)}\right) \leq D, \quad \text { for } n \text { sufficiently large, }
$$

and the fact that $R^{q c}(D)$ is a non-increasing function of $D$.

A natural choice for each $\Delta_{x}$ in the distortion observable in (10) is

$$
\Delta_{x}=I-|x\rangle\langle x| .
$$

For such a choice, the distortion of the classical data, resulting from the measurement, is measured with respect to the classical data that would result from an ideal measurement of the source state $\rho$ in its eigenbasis. However, such a choice is effectively classical because the operators $\Delta_{x}$ are diagonal in the Schmidt basis of $\psi_{R A}^{\rho}$. We show in Lemma 4 that, for such a choice of the distortion observable, the best strategy for rate-distortion coding amounts to an effectively classical strategy, in which Alice measures each output of the source state in its eigenbasis, thus obtaining a classical sequence, which she then compresses by applying the purely classical protocol for Shannon's rate-distortion coding. Thus, a necessary condition for there to be a quantum advantage in quantum-to-classical rate distortion coding is that the operators $\Delta_{x}$ should not be diagonal in the Schmidt basis of $\psi_{R A}^{\rho}$. After Lemma 4, we provide an example of a quantum source and a distortion observable for which quantum-to-classical rate distortion coding gives an advantage over the above classical strategy.

Lemma 4. If each operator $\Delta_{x}$, in the definition (10) of the distortion observable, is diagonal in the Schmidt basis of $\psi_{R A}^{\rho}$, then a quantum-to-classical rate distortion coding scheme has no advantage over a classical scheme, in the following sense: the optimal measurement map is a von Neumann measurement in the eigenbasis of the source state, followed by classical post-processing of the measurement result according to Shannon's rate distortion theory.

Proof. Let $\Lambda$ denote the minimal POVM in (15) for a given distortion $D$, and let $\mathcal{M}_{\Lambda}$ denote the corresponding measurement map. Let the Schmidt decomposition of the purification $\psi_{R A}^{\rho}$ of the source state $\rho \in \mathcal{D}\left(\mathcal{H}_{A}\right)$ be as follows:

$$
\left|\psi_{R A}^{\rho}\right\rangle=\sum_{z} \sqrt{p(z)}|z\rangle_{R}|z\rangle_{A}
$$


where $p(z)$ are the Schmidt coefficients. Then the distortion that the map $\mathcal{M}_{\Lambda}$ causes is as follows:

$$
\begin{aligned}
& \operatorname{Tr}\left\{\Delta_{R X}\left(\operatorname{id}_{R} \otimes \mathcal{M}_{\Lambda}\right)\left(\psi_{R A}^{\rho}\right)\right\} \\
& =\operatorname{Tr}\left\{\left(\sum _ { x } \Delta _ { x } \otimes | x \rangle \langle x | _ { X } ) \left(\sum_{z, z^{\prime}, y} \sqrt{p(z) p\left(z^{\prime}\right)}|z\rangle\left\langle\left. z^{\prime}\right|_{R} \otimes \operatorname{Tr}\left\{\Lambda_{y}|z\rangle\left\langle\left. z^{\prime}\right|_{A}\right\}|y\rangle\langle y|\right)\right\}\right.\right.\right. \\
& =\sum_{x, z, z^{\prime}} \sqrt{p(z) p\left(z^{\prime}\right)}\left\langle z^{\prime}\left|\Delta_{x}\right| z\right\rangle\left\langle z^{\prime}\left|\Lambda_{x}\right| z\right\rangle_{A},
\end{aligned}
$$

which is equivalent to

$$
\operatorname{Tr}\left\{\left(\sum_{x} \Delta_{x} \otimes \Lambda_{x}\right)\left(\psi_{R A}^{\rho}\right)\right\} .
$$

Now suppose that each $\Delta_{x}$ is diagonal in the Schmidt basis $\left\{|z\rangle_{R}\right\}$ of the reference system, so that

$$
\Delta(x)=\sum_{z}\left\langle z\left|\Delta_{x}\right| z\right\rangle_{R}|z\rangle\left\langle\left. z\right|_{R} .\right.
$$

Then the above expression for the distortion reduces to the following one:

$$
\sum_{x, z} p(z)\left\langle z\left|\Delta_{x}\right| z\right\rangle_{R}\left\langle z\left|\Lambda_{x}\right| z\right\rangle_{A}
$$

Consider $\mathcal{H}_{R} \simeq \mathcal{H}_{A}$ and choose $\left\{|z\rangle_{R}\right\}$ and $\left\{|z\rangle_{A}\right\}$ to be identical bases, which we simply denote as $\{|z\rangle\}$. Then (28) implies that, starting from the original POVM $\Lambda$, we can construct another POVM $\Lambda^{\prime}$ (say) which is diagonal in the eigenbasis of $\rho$, and which results in a distortion equal to that caused by the original POVM. The POVM $\Lambda^{\prime}$ is given by

$$
\Lambda^{\prime}:=\left\{\Lambda_{x}^{\prime}\right\}, \quad \text { where } \quad \Lambda_{x}^{\prime}:=\sum_{z}\left\langle z\left|\Lambda_{x}\right| z\right\rangle|z\rangle\langle z| .
$$

Clearly, the following identity holds

$$
\sum_{x, z} p(z)\left\langle z\left|\Delta_{x}\right| z\right\rangle\left\langle z\left|\Lambda_{x}\right| z\right\rangle=\sum_{x, z} p(z)\left\langle z\left|\Delta_{x}\right| z\right\rangle\left\langle z\left|\Lambda_{x}^{\prime}\right| z\right\rangle .
$$

The joint state of the reference system and the post-measurement classical register, resulting from the POVMs $\Lambda$ and $\Lambda^{\prime}$, are respectively given as follows:

$$
\sigma_{R X}=\left(\mathrm{id}_{R} \otimes \mathcal{M}_{\Lambda}\right)\left(\psi_{R A}^{\rho}\right), \quad \text { and } \quad \sigma_{R X}^{\prime}=\left(\mathrm{id}_{R} \otimes \mathcal{M}_{\Lambda^{\prime}}\right)\left(\psi_{R A}^{\rho}\right),
$$

where $\mathcal{M}_{\Lambda^{\prime}}$ is the measurement map corresponding to the POVM $\Lambda^{\prime}$. It turns out that the mutual information $I(X ; R)_{\sigma}$ can only be smaller than $I(X ; R)_{\sigma^{\prime}}$. This can be seen as follows. Note that we can equivalently write the state $\sigma_{R X}$ as

$$
\sigma_{R X}=\sum_{x}\left(\sqrt{\rho} \Lambda_{x}^{T} \sqrt{\rho}\right)_{R} \otimes|x\rangle\left\langle\left. x\right|_{X} .\right.
$$

Then the state $\sigma_{R X}^{\prime}$ can be written as

$$
\sigma_{R X}^{\prime}=\sum_{x}\left(\sqrt{\rho}\left(\sum_{z}\left\langle z\left|\Lambda_{x}^{T}\right| z\right\rangle|z\rangle\langle z|\right) \sqrt{\rho}\right)_{R} \otimes|x\rangle\left\langle\left. x\right|_{X} .\right.
$$

Since $\{|z\rangle\}$ is the eigenbasis of $\rho$, it follows that $[\sqrt{\rho},|z\rangle\langle z|]=0$, and hence the above state is equivalent to the following one:

$$
\sum_{x}\left(\sum_{z}\left\langle z\left|\left(\sqrt{\rho} \Lambda_{x}^{T} \sqrt{\rho}\right)\right| z\right\rangle|z\rangle\langle z|\right) \otimes|x\rangle\left\langle\left. x\right|_{X},\right.
$$

which is a classical-classical state. Note that such a state is equivalent to the state which would result from the action of a completely dephasing channel on the reference system $R$ of the state $\sigma_{R X}$ given 
by (30), i.e., $\sigma_{R X}^{\prime}=(\mathcal{N} \otimes$ id $) \sigma_{R X}$, where $\mathcal{N}$ denotes a completely dephasing channel. The mutual information can only decrease under such a map and hence $I(X ; R)_{\sigma^{\prime}} \leq I(X ; R)_{\sigma}$. This implies that in this case the optimal measurement to perform on the source is a von Neumann measurement in the eigenbasis of $\rho$, followed by classical post-processing according to the conditional distribution given by $p(x \mid z) \equiv\left\langle z\left|\Lambda_{x}\right| z\right\rangle$ (that this is a distribution follows from the fact that $\sum_{x} \Lambda_{x}=I$ ). Thus, this is equivalent to what one would obtain by exploiting Shannon's rate distortion theorem in a straightforward way.

The following example illustrates a scenario in which a quatum-to-classical rate distortion coding gives an advantage over a purely classical strategy.

Example: Consider a quantum information source which generates the states $|+\rangle$ and $|0\rangle$ with equal probability $1 / 2$, so that the density operator for the source is

$$
\rho=1 / 2(|+\rangle\langle+|+| 0\rangle\langle 0|)=\cos ^{2}(\pi / 8)\left|\phi_{0}\right\rangle\left\langle\phi_{0}\left|+\sin ^{2}(\pi / 8)\right| \phi_{1}\right\rangle\left\langle\phi_{1}\right|,
$$

where

$$
\begin{aligned}
& \left|\phi_{0}\right\rangle \equiv \cos (\pi / 8)|0\rangle+\sin (\pi / 8)|1\rangle, \\
& \left|\phi_{1}\right\rangle \equiv \sin (\pi / 8)|0\rangle-\cos (\pi / 8)|1\rangle .
\end{aligned}
$$

A purification of the source state $\rho$ is given by

$$
\left|\psi_{R A}^{\rho}\right\rangle=\cos (\pi / 8)\left|\phi_{0}\right\rangle_{R}\left|\phi_{0}\right\rangle_{A}+\sin (\pi / 8)\left|\phi_{1}\right\rangle_{R}\left|\phi_{1}\right\rangle_{A} .
$$

Suppose we are interested in measuring the distortion of the classical data (obtained as a result of a quantum-to-classical rate distortion task), by how much it deviates from the quantum states that specify the ensemble of the quantum information source. In this case, we would choose our distortion observable to be as follows:

$$
\Delta_{R X}=(I-|+\rangle\langle+|)_{R} \otimes|0\rangle\left\langle\left. 0\right|_{X}+(I-|0\rangle\langle 0|)_{R} \otimes \mid 1\right\rangle\left\langle\left. 1\right|_{X} .\right.
$$

Note that if we consider a two-outcome POVM $\Lambda=\left\{\Lambda_{0}, \Lambda_{1}\right\}$, where $\Lambda_{0}=I / 2=\Lambda_{1}$, then the state $\sigma_{R X}$ defined by (16) is given by

$$
\sigma_{R X}=\rho_{R} \otimes \frac{I}{2}
$$

where

$$
\rho_{R}=\operatorname{Tr}_{A}\left\{\psi_{R A}^{\rho}\right\}=\cos ^{2}(\pi / 8)\left|\phi_{0}\right\rangle\left\langle\left.\phi_{0}\right|_{R}+\sin ^{2}(\pi / 8) \mid \phi_{1}\right\rangle\left\langle\left.\phi_{1}\right|_{R} .\right.
$$

In this case, the choice (32) of the distortion observable yields the following value of the distortion:

$$
D \equiv \operatorname{Tr}\left\{\Delta_{R X}\left(\mathrm{id}_{R} \otimes \mathcal{M}_{\Lambda}\right)\left(\psi_{R A}^{\rho}\right)\right\}=1 / 4
$$

Moreover, since the state $\sigma_{R X}$ is uncorrelated, we have that $I(X ; R)_{\sigma}=0$, and hence, by Theorem 3, the rate distortion function $R^{q c}(D)$ is equal to zero. This implies that to obtain the full rate-distortion trade-off curve, one only needs to consider values of the distortion $D$ in the range $0 \leq D \leq 1 / 4$.

The rate-distortion trade-off curve, for the above range of values of $D$, was obtained numerically for the rate distortion observable defined by (32), and is given by the boundary of the shaded region in Fig. 2. As expected, the curve decreases monotonically with $D$.

To prove that in this case a quantum-to-classical rate distortion coding gives an advantage over a purely classical strategy, consider a two-outcome POVM $\Lambda$ which corresponds to a von Neumann measurement in the eigenbasis of the source state $\rho$, i.e., $\Lambda=\left\{\Lambda_{0}, \Lambda_{1}\right\}$, where

$$
\Lambda_{0}=\left|\phi_{0}\right\rangle\left\langle\phi_{0}\right| \quad \text { and } \quad \Lambda_{1}=\left|\phi_{1}\right\rangle\left\langle\phi_{1}\right|,
$$

or, more generally, consider any $\Lambda=\left\{\Lambda_{0}, \Lambda_{1}\right\}$ such that $\mathcal{N}\left(\Lambda_{i}\right)=\Lambda_{i}$ for $i=0,1$, where $\mathcal{N}$ denotes a dephasing channel, with the dephasing being in the eigenbasis of $\rho$. In this case one finds that, if the distortion observable is chosen as in (32), the distortion is always equal to the maximum 


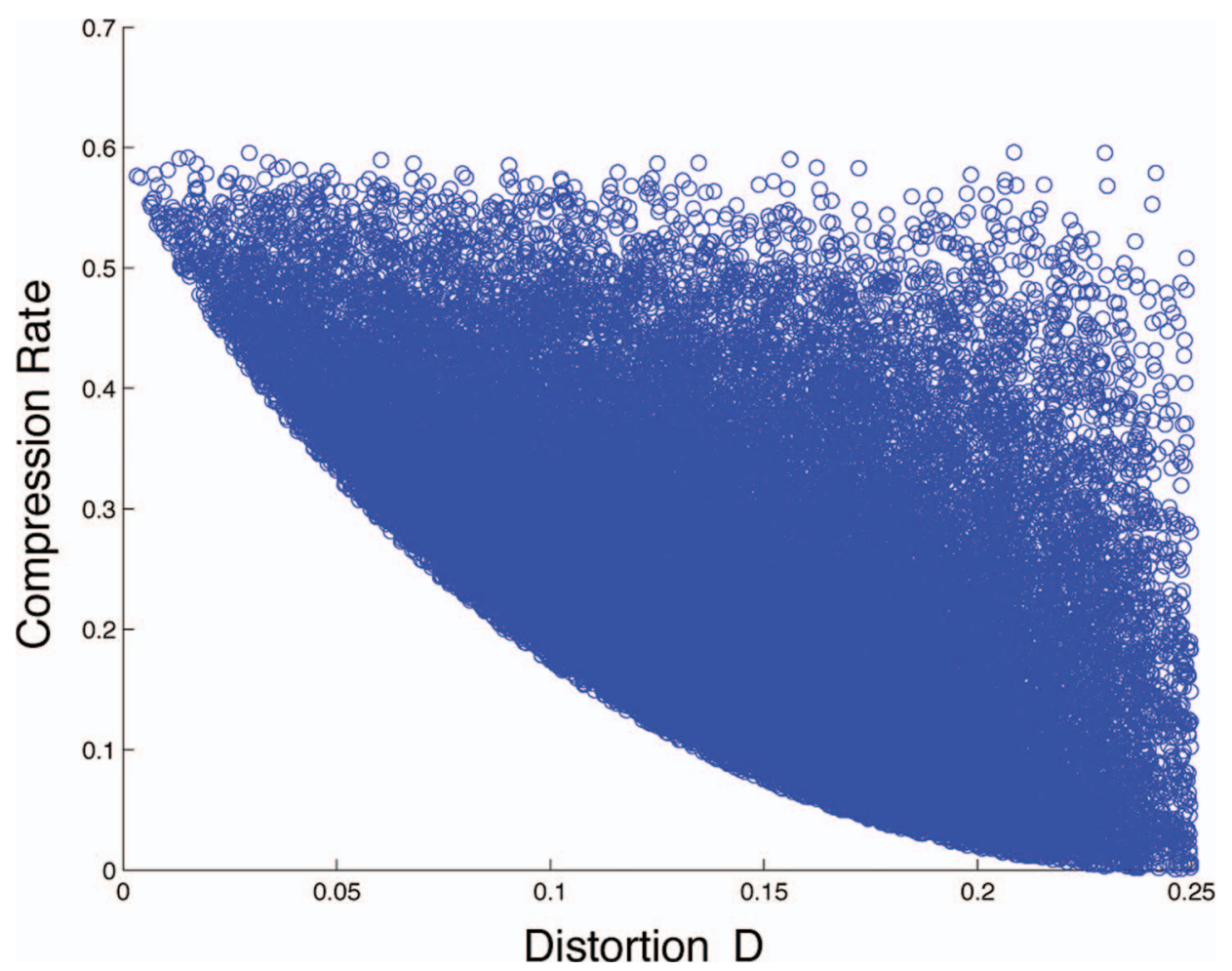

FIG. 2. A plot of compression rate vs. distortion for the quantum information source $\rho$ given by (31) and the rate distortion observable given by (32). It was obtained by randomly sampling 250000 two-outcome POVMs, and (for those POVMs which satisfy the distortion criterion $D \leq 1 / 4$ ) plotting the mutual information $I(X ; R)_{\sigma}$ for the resulting state $\sigma_{R X}$ (defined by (16)) against the corresponding value of the distortion. The boundary of the shaded region defines the rate-distortion trade-off curve.

allowed value $D=1 / 4$. This implies that for distortion in the range $0 \leq D<1 / 4$, for the choice (32), quantum-to-classical rate-distortion coding gives an advantage over a classical strategy. ${ }^{28}$

\section{QUANTUM-TO-CLASSICAL RATE-DISTORTION CODING WITH QUANTUM SIDE INFORMATION}

We now consider a class of protocols in which Alice and Bob share many copies of some quantum state $\rho_{A B}$. This state can be considered to arise from the action of an isometry on the state of a memoryless quantum information source performed by a third party (say, Charlie), who then distributes the systems $A$ and $B$ to Alice and Bob, respectively. The system $B$ acts as Bob's quantum side information. We also let Alice and Bob share common randomness. The goal is to quantify the minimum rate at which Alice needs to send classical data to Bob, such that he can reconstruct a classical description of the state $\rho_{A}=\operatorname{Tr}_{B}\left\{\rho_{A B}\right\}$ by using the received classical data and his quantum side information. By a "classical description," we mean that for a fixed distortion $D \geq 0$, where the distortion is defined as

$$
d\left(\rho, \mathcal{M}_{\Lambda}\right) \equiv \operatorname{Tr}\left\{\Delta_{R X B}\left(\mathrm{id}_{R} \otimes \mathcal{M}_{\Lambda} \otimes \mathrm{id}_{B}\right)\left(\psi_{R A B}^{\rho}\right)\right\},
$$

and a chosen distortion observable $\Delta$ of the following form:

$$
\Delta_{R B X} \equiv \sum_{x} \Delta_{R B}^{x} \otimes|x\rangle\left\langle\left. x\right|_{X},\right.
$$

we require that (13) is satisfied. The rate distortion function in this scenario is defined in a manner analogous to $R^{q c}(D)$ of Sec. IV and is denoted as $R_{q s i}^{q c}(D)$. In the above, $\psi_{R A B}^{\rho}$ is a purification of the state $\rho_{A B}$, and since we are interested in measuring the distortion that occurs on the $A$ system only, the 


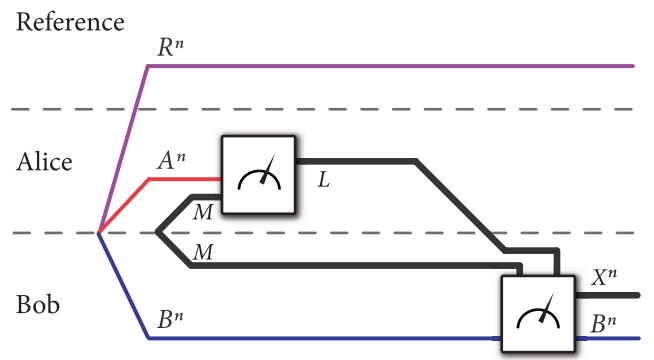

FIG. 3. The most general protocol for quantum-to-classical rate-distortion coding with quantum side information. Alice and Bob share many copies of a quantum state $\rho_{A B}$, which is purified by an inaccessible reference system. We also allow them access to common randomness $M$ before the protocol begins. Alice first performs a collective measurement on her systems, producing a classical output $L$. She then transmits $L$ over noiseless classical bit channels to Bob. Bob performs a collective measurement on his quantum systems, depending on what he receives from Alice and his share of the common randomness. This measurement produces a classical sequence $X^{n}$ and has quantum outputs as well. The protocol is deemed successful if the classical sequence $X^{n}$ is not distorted on average from the quantum source more than a specified amount according to a suitable distortion observable. We also demand that the disturbance caused by the protocol to the joint state of the reference and Bob's systems is asymptotically negligible. This in turn implies that quantum side information suffers a negligible disturbance and hence is available to Bob for future use.

operators $\Delta_{R B}^{x}$ in (34) should act on all systems that purify the $A$ system. Figure 3 depicts the most general protocol for quantum-to-classical rate-distortion coding with quantum side information.

Reference 22 contains a theorem that determines the optimal rates for measurement compression in the presence of quantum side information. It almost immediately leads to the following rate distortion theorem:

Theorem 5. For a memoryless quantum information source characterized by a state $\rho_{A B}$ (where Alice possesses $A$ and Bob possesses B), a quantum-to-classical distortion observable $\Delta_{R B X}$, and any given distortion $D \geq 0$, an achievable rate for quantum-to-classical rate distortion with quantum side information, when sufficient common randomness is available, is given by

$$
\min _{\Lambda: d\left(\rho, \mathcal{M}_{\Lambda}\right) \leq D} I(X ; R \mid B)_{\sigma},
$$

so that

$$
R_{q s i}^{q c}(D) \leq \min _{\Lambda: d\left(\rho, \mathcal{M}_{\Lambda}\right) \leq D} I(X ; R \mid B)_{\sigma},
$$

where $\Lambda \equiv\left\{\Lambda_{x}\right\}$ is a POVM acting only on Alice's system, $d\left(\rho, \mathcal{M}_{\Lambda}\right)$ is defined through (33)-(34), and $\psi_{R A B}^{\rho}$ is a purification of the state $\rho_{A B}$. The state $\sigma$ is the following classical-quantum state:

$$
\sigma_{X R B} \equiv \sum_{x}|x\rangle\left\langle\left. x\right|_{X} \otimes \operatorname{Tr}_{A}\left\{\left(I_{R} \otimes \Lambda_{x} \otimes I_{B}\right)\left(\psi_{R A B}^{\rho}\right)\right\} .\right.
$$

Proof. The proof of the achievability part of this theorem proceeds similarly to that of Theorem 3. We merely fix the POVM that minimizes the RHS of (35). From this POVM, we can construct a protocol for measurement compression with quantum side information by invoking Theorem 12 of Ref. 22. This protocol exploits classical communication at a rate $I(X ; R \mid B)_{\sigma}$ and common randomness at a rate $H(X \mid R B)_{\sigma}$ in order to simulate the action of the POVM on many copies of the state $\rho_{A B}$. By an argument similar to that in the proof of the achievability part of Theorem 3, we know that such a protocol meets the distortion criterion and that it is possible to derandomize the common randomness in the same way as in (22).

If, in addition, we demand that the protocol causes asymptotically negligible disturbance of the state of Bob (i.e., the quantum side information) and the state of the reference system, then we can prove that the upper bound in (36) is achieved. Hence in this case the rate distortion function is given by a single-letter formula. The requirement of the protocol leaving the states of Bob and the reference essentially undisturbed might seem somewhat restrictive at first. However, it can be 
justified as follows. Firstly, note that ignoring the quantum side information leads to a protocol with a classical communication rate of $I(X ; R B)$ which of course does not disturb the systems of the reference and Bob in any way. Secondly, Bob might wish to use the quantum side information in some future information-processing task, which therefore leads to the above requirement on the state of his system. In light of this, it seems reasonable to restrict consideration to a class of protocols in which Bob is allowed to exploit the quantum side information, but only in a way which causes negligible disturbance to it. These considerations yield the following theorem.

Theorem 6. For a memoryless quantum information source $\rho_{A B}$ (where Alice possesses $A$ and Bob possesses $B$ ), a quantum-to-classical distortion observable $\Delta_{R B X}$, and any given distortion $D$ $\geq 0$, the quantum-to-classical rate distortion function with quantum side information, sufficient common randomness, and such that the protocol causes only a negligible disturbance to the systems of the reference and Bob, is given by

$$
R_{q s i}^{q c}(D)=\min _{\Lambda: d\left(\rho, \mathcal{M}_{\Lambda}\right) \leq D} I(X ; R \mid B)_{\sigma},
$$

where the state $\sigma$ is as defined in (37) of Theorem 5.

Proof. The proof of the achievability part of this theorem again follows directly from Theorem 12 of Ref. 22 which deals with measurement compression in the presence of quantum side information. We merely fix the map that minimizes the expression in (38) and apply the aforementioned theorem. The resulting protocol meets the distortion constraint because of the way that the POVM is chosen in (38).

The converse part of this theorem exploits the approach from the converse parts of Theorems 12 and 14 of Ref. 22, which in turn exploit ideas of Cuff. ${ }^{7}$ The most general protocol begins with the state $\left(\psi_{R A B}^{\rho}\right)^{\otimes n}$ shared between the reference, Alice, and Bob. We let Alice and Bob share common randomness as well (embodied in some random variable $M$ ). Alice performs an encoding on her systems $A^{n}$ with the help of her share of the common randomness $M$, producing a classical output given by the random variable $L$ which takes values in a finite alphabet $\mathcal{L}$. Let $\sigma$ denote the state at this point. Also, let $R$ be the rate of classical communication, i.e., $R=\left(\log _{2}|\mathcal{L}|\right) / n$. Alice sends $L$ to Bob, who then combines this with his share of the common randomness to perform some decoding map on $B^{n}$, producing a classical sequence $X^{n}$ and a quantum system $B^{\prime n}$. Let $\omega$ denote the final state after this encoding-decoding procedure. Further, let $\mathcal{F}_{n}^{A^{n} B^{n} \rightarrow X^{n} B^{\prime n}}$ denote the effective CPTP map on $\rho_{A B}^{\otimes n}$ (the state that Alice and Bob share at the start of the protocol) resulting from these encoding and decoding operations. We demand that the distortion of the output $X^{n}$ be no larger than $D$ (in a sense similar to that in (23), though in this case we need to trace over the systems $B^{\prime n}$ ), and we furthermore demand that the trace distance between $\left(\psi_{R B}^{\rho}\right)^{\otimes n}$ and the state $\omega_{R^{n} B^{\prime n}}$ on systems $R^{n} B^{\prime n}$ (at the end of the protocol) be no larger than some arbitrarily small $\varepsilon>0$. The converse then proceeds as follows. For $n$ large enough,

$$
\begin{aligned}
n R & \geq H(L)_{\sigma} \\
& \geq I\left(L ; M B^{n} R^{n}\right)_{\sigma} \\
& =I\left(L M B^{n} ; R^{n}\right)_{\sigma}+I\left(L ; M B^{n}\right)_{\sigma}-I\left(R^{n} ; B^{n} M\right)_{\sigma} \\
& \geq I\left(L M B^{n} ; R^{n}\right)_{\sigma}-I\left(R^{n} ; B^{n}\right)_{\sigma} \\
& \geq I\left(X^{n} B^{\prime n} ; R^{n}\right)_{\omega}-I\left(R^{n} ; B^{\prime n}\right)_{\omega}-n \varepsilon^{\prime} \\
& \geq \sum_{k}\left[I\left(X_{k} B_{k}^{\prime} ; R_{k}\right)_{\omega}-I\left(R_{k} ; B_{k}^{\prime}\right)\right]-2 n \varepsilon^{\prime} \\
& =\sum_{k} I\left(X_{k} ; R_{k} \mid B_{k}^{\prime}\right)_{\omega}-2 n \varepsilon^{\prime} .
\end{aligned}
$$

The first inequality follows because the entropy of a system is always less than the logarithm of its dimension. The second inequality follows because $I\left(L ; M B^{n} R^{n}\right)_{\sigma}=H(L)_{\sigma}-H\left(L \mid M B^{n} R^{n}\right)_{\sigma}$ and 
$H\left(L \mid M B^{n} R^{n}\right)_{\sigma} \geq 0$ for a classical $L$. The first equality is an identity for quantum mutual information. The third inequality follows because the common randomness $M$ is in a product state with $R^{n} B^{n}$ so that $I\left(R^{n} ; B^{n} M\right)_{\sigma}=I\left(R^{n} ; B^{n}\right)_{\sigma}$ and because $I\left(L ; M B^{n}\right)_{\sigma} \geq 0$. The fourth inequality follows from quantum data processing, Lemma 1, (the systems $L M B^{n}$ are processed to produce systems $X^{n} B^{\prime n}$ ), and from the requirement that the protocol causes negligible disturbance of the state of $R^{n} B^{n}$. The term $\varepsilon^{\prime}$ (which is a function of $\varepsilon$ ) arises from an application of the Alicki-Fannes' inequality, ${ }^{1}$ where $\lim _{\varepsilon} \rightarrow 0 \varepsilon^{\prime}(\varepsilon)$ $=0$. The fifth inequality follows from superadditivity of quantum mutual information (Lemma 2) and because the state on $R^{n} B^{\prime n}$ is close in trace distance to a tensor-product state (see Lemma 10 of Ref. 22). The second equality follows from the identity $I\left(X_{k} B_{k}^{\prime} ; R_{k}\right)_{\omega}-I\left(R_{k} ; B_{k}^{\prime}\right)$ $=I\left(X_{k} ; R_{k} \mid B_{k}^{\prime}\right)_{\omega}$.

At this point, we have argued that the above lower bound holds for a protocol that exploits

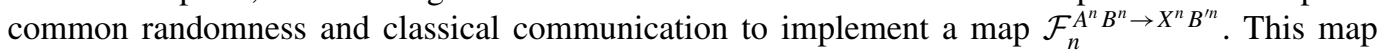
meets the distortion constraint while also causing only a negligible disturbance to the state on $R^{n} B^{n}$, in the sense that

$$
\left\|\operatorname{Tr}_{X^{n}}\left\{\mathcal{F}_{n}^{A^{n} B^{n} \rightarrow X^{n} B^{\prime n}}\left(\left(\psi_{R A B}^{\rho}\right)^{\otimes n}\right)\right\}-\left(\psi \rho_{R B}\right)^{\otimes n}\right\|_{1} \leq \varepsilon
$$

As in the proof of Theorem 14 of Ref. 22, applying Uhlmann's theorem to the above condition guarantees that there is some map acting only on Alice's system, such that the information quantity in the last line of the above chain of inequalities (39) does not change too much. For completeness, we repeat the argument here. Let the Kraus representation of $\mathcal{F}_{n}^{A^{n} B^{n} \rightarrow X^{n} B^{\prime n}}$ be given by

$$
\mathcal{F}_{n}^{A^{n} B^{n} \rightarrow X^{n} B^{\prime n}}(\cdot)=\sum_{i} F_{i}(\cdot) F_{i}^{\dagger} .
$$

A purification of $\operatorname{Tr}_{X^{n}}\left\{\mathcal{F}_{n}^{A^{n} B^{n} \rightarrow X^{n} B^{\prime n}}\left(\left(\psi_{R A B}^{\rho}\right)^{\otimes n}\right)\right\}$ is given by

$$
\sum_{i} F_{i}\left(\left|\psi_{R A B}^{\rho}\right\rangle\right)^{\otimes n} \otimes|i\rangle_{I},
$$

where $I$ is a purifying system, while a purification of $\left(\psi_{R B}^{\rho}\right)^{\otimes n}$ is $\left(\left|\psi_{R A B}^{\rho}\right\rangle\right)^{\otimes n}$. By Uhlmann's theorem, there is an isometry $U^{A^{n} \rightarrow X^{n} I}$ acting only on Alice's system, taking $\left(\left|\psi_{R A B}^{\rho}\right\rangle\right)^{\otimes n}$ to an approximation of the state in (40) such that the trace distance between this state and $U^{A^{n} \rightarrow X^{n} I}\left(\left|\psi_{R A B}^{\rho}\right\rangle\right)^{\otimes n}$ is at most $2 \sqrt{\varepsilon}$. Thus, the map on Alice's side consists of applying $U^{A^{n} \rightarrow X^{n} I}$ and tracing out $I$. Let $\omega^{\prime}$ denote the resulting state. By exploiting this map instead of the original one, we find the following lower bound on the information quantity in (39):

$$
\sum_{k} I\left(X_{k} ; R_{k} \mid B_{k}\right)_{\omega^{\prime}}-3 n \varepsilon^{\prime} .
$$

The important feature of this approximation map is that it acts only on Alice's side. Continuing, we have

$$
\begin{aligned}
& \geq \sum_{k} R_{q s i}^{q c}\left(d\left(\rho, \mathcal{G}_{n}^{(k)}\right)\right)-3 n \varepsilon^{\prime} \\
& =n \sum_{k} \frac{1}{n} R_{q s i}^{q c}\left(d\left(\rho, \mathcal{G}_{n}^{(k)}\right)\right)-3 n \varepsilon^{\prime} \\
& \geq n R_{q s i}^{q c}\left(\sum_{k} \frac{1}{n} d\left(\rho, \mathcal{G}_{n}^{(k)}\right)\right)-3 n \varepsilon^{\prime} \\
& \geq n R_{q s i}^{q c}(D)-3 n \varepsilon^{\prime} .
\end{aligned}
$$

In the above, $\mathcal{G}_{n}^{(k)}$ is the marginal operation on the $k$ th copy of the source space induced by the overall encoding and approximation of the decoding guaranteed by Uhlmann's theorem. The first inequality follows from the fact that the map $\mathcal{G}_{n}^{(k)}$ has distortion $d\left(\rho, \mathcal{G}_{n}^{(k)}\right)$ and the expression (38) for the rate-distortion function $R_{q s i}^{q c}$ in Theorem 6 involves a minimum over all maps on Alice's 
system with this distortion. The first equality is obvious. The last two inequalities follow because the rate-distortion function is convex and non-increasing as a function of $D$ (the proof of convexity is similar to the proof of Lemma 14 of Ref. 8, though here we rely on the map acting solely on Alice's system).

A special case of the above theorem is the setting considered in Theorem 4.2 of Ref. 13. There, Luo and Devetak considered the scenario in which the source is a classical-quantum state of the form:

$$
\sum_{y} p_{Y}(y)|y\rangle\left\langle\left. y\right|_{Y} \otimes \rho_{B}^{y}\right.
$$

where Alice possesses $Y$ and Bob $B$. The goal is for Alice to transmit her classical data to Bob up to some distortion, and Bob is allowed to use the quantum side information to help reduce the communication costs. They proved that the following rate is achievable:

$$
\min _{p_{X \mid Y}(x \mid y): \mathbb{E}\{d(x, y)\} \leq D} I(X ; Y \mid B)_{\sigma},
$$

for some classical distortion measure $d(x, y)$ and where the information quantity is with respect to a state of the following form:

$$
\sum_{y} p_{X \mid Y}(x \mid y) p_{Y}(y)|y\rangle\left\langle\left. y\right|_{Y} \otimes \mid x\right\rangle\left\langle\left. x\right|_{X} \otimes \rho_{B}^{y} .\right.
$$

Luo and Devetak were not able to find a single-letter characterization of the rate-distortion function, but with our additional assumptions of sufficient common randomness and a negligible disturbance of the quantum side information, our theorem reduces to a single-letter characterization for their setting. In fact, if one chooses the distortion observable in (34) so that the operators $\Delta_{R B}^{x}$ are diagonal in the Schmidt basis of the $R B$ systems of $\psi_{R A B}^{\rho}$, then a similar statement as in Lemma 4 applies. That is, in this case, it is optimal to measure the $A$ system in the eigenbasis of $\rho_{A}$ and proceed according to the protocol of Luo and Devetak in Ref. 13. As stated above, their protocol is optimal if we demand that it cause only a negligible disturbance to the state of the reference and Bob.

\section{CONCLUSIONS AND DISCUSSIONS}

We have derived a single-letter formula for the quantum-to-classical rate distortion function. The goal in quantum-to-classical rate-distortion coding is to provide a compressed classical description of a quantum source, up to some specified level of distortion, as determined by a distortion observable. The formula is expressed as a minimization of a quantum mutual information over all quantum-toclassical channels that meet the distortion constraint. In general, our results show that a collective measurement of the quantum source is required to obtain optimal compression rates. However, if the distortion observable has a classical form (so that each operator $\Delta_{x}$ is diagonal in the Schmidt basis), then the best strategy for quantum-to-classical rate-distortion coding ends up being an effectively classical strategy, in which Alice performs individual measurements of each copy of the source in its eigenbasis, and processes the resulting classical data according to Shannon's classical rate-distortion protocol.

We have also derived a single-letter formula for the quantum-to-classical rate distortion function when the receiver has some quantum side information about the source. Our assumptions are that Alice and Bob share sufficient common randomness, and that the protocol causes only a negligible disturbance to the joint state of the reference and the quantum side information. We consider this latter assumption to be rather natural, since Bob might wish to make use of his quantum side information in some future protocol. Our results suggest that it might generally be possible for quantum information-theoretic protocols that employ quantum side information to be simplified by employing this assumption, due to the restriction that it imposes on the quantum states at the output 
of a given protocol. This assumption is purely non-classical, since it is always possible to copy classical information before processing it in any way.

There are some interesting open questions to consider going forward from here. It would be ideal if we could derandomize the common randomness in the protocol that uses quantum side information, since it would imply that this extra resource is unnecessary. However, if we did so, the protocol for measurement compression with quantum side information could end up causing a nonnegligible disturbance to the joint state of the reference and Bob's systems, for some of the values of the common randomness. Since our approach in the proof of the achievability part of the coding theorem relies on this protocol, we have not been able to conclude that the common randomness is unnecessary. However, the common randomness plays only a passive role in the converse theorem, and this suggests that it might ultimately be unnecessary. In order to determine if this is the case, one would have to consider a different protocol in proving the achievability part of the coding theorem.

\section{ACKNOWLEDGMENTS}

We acknowledge useful discussions Patrick Hayden. M.M.W. acknowledges support from the Centre de Recherches Mathématiques at the University of Montreal. M.-H.H. received support from the Chancellor's postdoctoral research fellowship, University of Technology Sydney (UTS) and was also partly supported by the National Natural Science Foundation of China (Grant No. 61179030) and the Australian Research Council (Grant No. DP120103776). AW was supported by the Royal Society, the Philip Leverhulme Trust, EC integrated project QAP (contract IST-2005-15848), the STREPs QICS and QCS, and the ERC Advanced Grant "IRQUAT".

${ }^{1}$ R. Alicki and M. Fannes, "Continuity of quantum conditional information," J. Phys. A 37(5), L55-L57 (2004).

${ }^{2}$ H. Barnum, "Quantum rate-distortion coding," Phys. Rev. A 62(4), 042309 (2000).

${ }^{3}$ T. Berger, Rate Distortion Theory: A Mathematical Basis for Data Compression, Information and System Sciences Series (Prentice Hall, 1971).

${ }^{4}$ M. Berta, O. Fawzi, and S. Wehner, "Quantum to classical randomness extractors," Lect. Notes Comput. Sci. 7417, 776-793 (2012).

${ }^{5}$ X.-Y. Chen and W.-M. Wang, "Entanglement information rate distortion of a quantum Gaussian source," IEEE Trans. Inf. Theory 54(2), 743-748 (2008).

${ }^{6}$ T. M. Cover and J. A. Thomas, Elements of Information Theory, 2nd ed. (Wiley-Interscience, 2005).

${ }^{7}$ P. Cuff, "Communication requirements for generating correlated random variables," in Proceedings of the $2008 \mathrm{In}$ ternational Symposium on Information Theory, Toronto, Ontario, Canada, July 2008 (Stanford Univ., Stanford, CA), pp. 1393-1397; e-print arXiv:0805.0065.

${ }^{8}$ N. Datta, M.-H. Hsieh, and M. M. Wilde, "Quantum rate distortion, reverse Shannon theorems, and source-channel separation,” IEEE Trans. Inf. Theory 59, 615-630 (2013); e-print arXiv:1108.4940.

${ }^{9}$ I. Devetak and T. Berger, "Quantum rate-distortion theory for memoryless sources," IEEE Trans. Inf. Theory 48(6), 1580-1589 (2002); e-print arXiv:quant-ph/0011085.

${ }^{10}$ I. Devetak and A. Winter, "Classical data compression with quantum side information," Phys. Rev. A 68(4), 042301 (2003).

${ }^{11}$ V. Giovannetti, S. Guha, S. Lloyd, L. Maccone, J. H. Shapiro, and H. P. Yuen, "Classical capacity of the lossy bosonic channel: The exact solution," Phys. Rev. Lett. 92(2), 027902 (2004).

${ }^{12}$ A. S. Holevo, "The capacity of the quantum channel with general signal states," IEEE Trans. Inf. Theory 44, 269-273 (1998).

${ }^{13}$ Z. Luo and I. Devetak, "Channel simulation with quantum side information," IEEE Trans. Inf. Theory 55(3), 1331-1342 (2009); e-print arXiv:quant-ph/0611008.

${ }^{14}$ M. A. Nielsen and I. L. Chuang, Quantum Computation and Quantum Information (Cambridge University Press, 2000).

${ }^{15}$ B. Schumacher, "Quantum coding," Phys. Rev. A 51(4), 2738-2747 (1995).

${ }^{16}$ B. Schumacher and M. A. Nielsen, "Quantum data processing and error correction," Phys. Rev. A 54, 2629-2635 (1996).

${ }^{17}$ B. Schumacher and M. D. Westmoreland, "Sending classical information via noisy quantum channels," Phys. Rev. A 56(1), 131-138 (1997).

${ }^{18}$ C. E. Shannon, “A mathematical theory of communication,” Bell Syst. Tech. J. 27, 379-423 (1948).

${ }^{19}$ C. E. Shannon, "Coding theorems for a discrete source with a fidelity criterion," IRE Int. Conv. Rec. 7, 142-163 (1959).

${ }^{20}$ D. Slepian and J. K. Wolf, “Noiseless coding of correlated information sources,” IEEE Trans. Inf. Theory 19(4), 471-480 (1973).

${ }^{21}$ M. M. Wilde, "From Classical to Quantum Shannon Theory," preprint arXiv:1106.1445 (2011).

${ }^{22}$ M. M. Wilde, P. Hayden, F. Buscemi, and M.-H. Hsieh, "The information-theoretic costs of simulating quantum measurements,” J. Phys. A: Math. Theor. 45(45), 453001 (2012); e-print arXiv:1206.4121. 
${ }^{23}$ A. Winter, "Compression of sources of probability distributions and density operators," preprint arXiv:quant-ph/0208131 (2002).

${ }^{24} \mathrm{~A}$. Winter, "“Extrinsic" and "intrinsic" data in quantum measurements: asymptotic convex decomposition of positive operator valued measures," Commun. Math. Phys. 244(1), 157-185 (2004).

${ }^{25}$ A. Winter and R. Ahlswede, "Quantum rate-distortion theory," (unpublished).

${ }^{26} \mathrm{~A}$. Wyner and J. Ziv, "The rate-distortion function for source coding with side information at the decoder," IEEE Trans. Inf. Theory 22(1), 1-10 (1976).

${ }^{27}$ We note that a similar perspective was used to justify the development of quantum-to-classical randomness extractors. ${ }^{4}$

${ }^{28} \mathrm{~A}$ "classical strategy" here corresponds to a measurement in the eigenbasis of $\rho$, followed by classical post-processing. 


\section{Fwd: Your manuscript \#13-0102 accepted for publication in JMP}

Mark M. Wilde <mwilde@gmail.com>

Mon, Mar 11, 2013 at 3:44 PM

To: Nilanjana Datta <n.datta@statslab.cam.ac.uk>, Andreas Winter <der.winter@googlemail.com>, Min-Hsiu Hsieh $<$ minhsiuh@gmail.com>

All,

Good news for our "quantum to classical rate distortion" submission to JMP: It was accepted without request for any changes. This could of course mean one (or both) of two things: 1) Our paper is fabulous :) or 2) the referee was lazy. In any case, I'm glad that we received an accept. I guess it will be a very long time before we hear back from IEEE on our other paper. talk to you soon,

mark

---- Forwarded message

From: <jmp@math.ucdavis.edu>

Date: Mon, Mar 11, 2013 at 4:37 PM

Subject: Your manuscript \#13-0102 accepted for publication in JMP

To: mwilde@gmail.com

Dear Dr. Wilde:

I am pleased to inform you that your manuscript \#13-0102, "Quantum-to-classical rate distortion coding" has been accepted for publication in the Journal of Mathematical Physics. Your manuscript has been tentatively scheduled to appear in the April issue.

The Journal of Mathematical Physics is published by the American Institute of Physics. In any publication correspondence you may have with their Journal Production Division (e.g. regarding the correction of proofs), please identify your paper by its author, title, journal (JMP) and log number. Contact them directly at:

\section{Editorial Supervisor}

JMP

AIP Journal Production Division

Suite 1 N01.

2 Huntington Quadrangle

Melville, NY 11747-4502

VOICE: (516) 576-2240

FAX: (631) 396-0060

E-MAIL: jmp@aip.org

\section{THE STATUS OF YOUR MANUSCRIPT}

During the production process, authors may access information about their accepted manuscript by visiting the AMSIS website at:

http://www.aip.org/msinq/status.html.

Please allow at least three business days after receiving this message before checking the manuscript status.

\section{PROOFS}

You will receive your proofs via the World Wide Web. Also, the JMP Editorial Office will automatically receive a copy in order to be better able to assist you if questions arise regarding your proofs, either in conjunction with or in addition to the AIP production office. After your paper has been composed, the proofs will be placed in a secure portion of the AIP server.

You will be notified of the location of your proofs by email. Please watch for this email message: the sender will be 
<jmp@aip.org>, and the subject line will read "This is your only AUTHOR PROOF for JMP".

This message will provide you with a Personal Identification Number (PIN) and the instructions for downloading your proofs. You will need Adobe's Acrobat Reader installed in order to view and print the proofs.

\section{NOTICE OF ACCEPTANCE}

Please note that this is the only official notice of acceptance of your manuscript you will receive. Should you need a hard copy of the acceptance letter, please let us know as soon as possible.

\section{REPRINTS}

A link to the Rightslink service for ordering reprints will be provided when proofs are ready for review.

We would also appreciate being informed of any changes in your mailing address, phones (direct, dept., and fax), or e-mail.

Sincerely,

Bruno Nachtergaele, Editor

Manuscript \#13-0102:

Editor's Recommendation: Publish as is

Associate Editor Recommendation: Publish as is

Referee Recommendation: Publish as is

Referee (Comments to the Author):

The paper deals with the lossy compression of the outcomes of the measurement performed by Alice and sent to Bob. It should be an important paper on this topic. The paper also deals with the effect of side information. The paper deals comprehensively with the topic with detailed study of prior works and when and how their results are more general than the classical results. This is important since we are looking at quantum to classical rate distortion. The paper is very well written and should be surely published by your journal.

\section{||||||||||||||||||||||||||||||||||}

Journal of Mathematical Physics

University of California at Davis

3125 Mathematical Sciences

Davis, CA 95616

United States of America

voice:(530) 752-0268

email: jmp@math.ucdavis.edu

URL: http://ojps.aip.org/jmp/

|||||||||||||||||||||||||||||||||| $\mid$

Mark M. Wilde

mwilde@gmail.com

web site: http://www.markwilde.com 
To: "Mark M. Wilde" <mwilde@gmail.com>

Cc: Nilanjana Datta <n.datta@statslab.cam.ac.uk>, Min-Hsiu Hsieh <minhsiuh@gmail.com>

Good news, a bit scary of course this rapidity, but indeed...who cares as

long as we appear :-P

- Andreas.

[Quoted text hidden]

$-$

Andreas Winter <der.winter@gmail.com>

ICREA +++ Fisica Teorica: Informacio i Fenomens Quantics,

Universitat Autonoma de Barcelona, ES-08193 Bellaterra (Barcelona).

Office C7b-036 // tel +34 93 581-1305 // fax 581-1938

mobile

Espanya +34610084828 . UK +44(0)7930906649. Italia +393462490981

Deutschland $+49(0) 1731360934 \ldots \ldots$. . Singapore +6590383633

Nilanjana Datta <n.datta@statslab.cam.ac.uk>

Mon, Mar 11, 2013 at 4:11 PM

To: "Mark M. Wilde" <mwilde@gmail.com>

Cc: Andreas Winter <der.winter@googlemail.com>, Min-Hsiu Hsieh <minhsiuh@gmail.com>

Yes...it's a bit disconcerting to receive such a short report after all the effort that we put in. Did the referee (only one!) really read it.. I wonder...

Anyway, I think we tried to be very thorough and hopefully there were no mistakes.

So, we should be glad!

Nilanjana

[Quoted text hidden]

Mark M. Wilde <mwilde@gmail.com>

Mon, Mar 11, 2013 at 4:11 PM

To: Andreas Winter <der.winter@googlemail.com>

Cc: Nilanjana Datta <n.datta@statslab.cam.ac.uk>, Min-Hsiu Hsieh <minhsiuh@gmail.com>

All,

There are two things I've been meaning to point out since we finished this q-c work (and I cannot remember if I have yet mentioned these):

1) The q-c paradigm for rate distortion might be a good way to explore if there are alternative approaches to quantum rate distortion besides the reverse Shannon theorem. That is, given a source rho, and a measurement $\{$ Lambda_y\} that optimizes the mutual information, we would generate codewords according to Tr\{Lambda_y rho\} (a number of them no smaller than an exponential in the mutual information of the source and the output of the measurement). For operation of the code, the idea would be to do a collective measurement on the source, obtain some sequence, and then send the closest codeword as the reconstruction. I haven't tried hard to figure out if a strategy like this would work, but it seems possible. As you might realize, it's very close to the standard classical strategy.

2) We forgot about doing c-q rate distortion. You might remember our discussion at McGill back in December 2011 when Andreas showed us how it doesn't make sense to do it, given a particular distortion measure that is essentially of the c-c type. Though, you might remember that our q-c project was in peril, until we realized to just change the distortion observable so that it is truly of the q-c type. I think it's clear that we could do a very similar thing for $\mathrm{c}-\mathrm{q}$ rate distortion and then obtain results that are interesting. This setting is also another one where we might figure out a strategy that is different from the reverse Shannon theorem.

I'd be curious to hear any of your thoughts on the above. talk to you soon,

mark

[Quoted text hidden] 


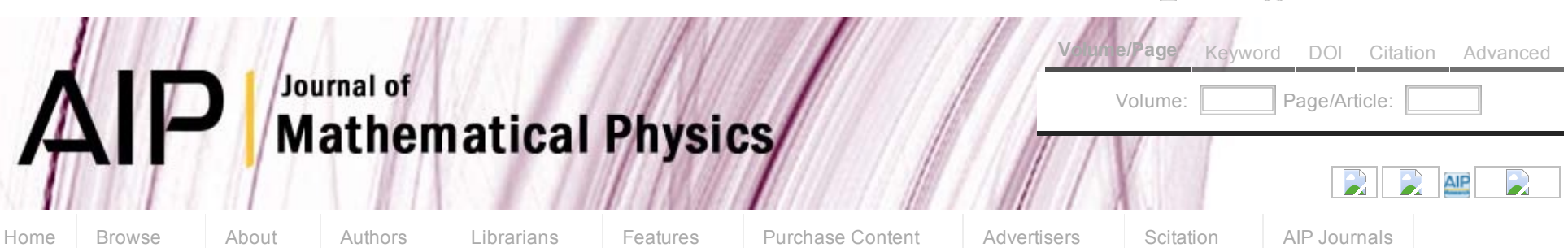

Journal of Mathematical Physics / Volume 54 / Issue 4 / ARTICLES / Quantum Information and Computation

Previous Article | Next Article

J. Math. Phys. 54, 042201 (2013); http://dx.doi.org/10.1063/1.4798396 (17 pages)

CrossMark

\& click for updates

\section{Quantum-to-classical rate distortion coding}

Nilanjana Datta ${ }^{1}$, Min-Hsiu Hsieh ${ }^{2}$, Mark M. Wilde ${ }^{3}$, and Andreas Winter ${ }^{4}$

${ }^{1}$ Statistical Laboratory, University of Cambridge, Wilberforce Road, Cambridge CB3 OWB, United Kingdom

${ }^{2}$ Centre for Quantum Computation \& Intelligent Systems, Faculty of Engineering and Information Technology, University of Technology, Sydney, P.O. Box 123, Broadway NSW 2007, Australia

${ }^{3}$ School of Computer Science, Mc Gill University, Montreal, Quebec H3A 2A7, Canada

${ }^{4}$ ICREA - Institució Catalana de Recerca i Estudis Avançats, Pg. Lluis Companys 23, ES-08010 Barcelona, Spain; Física Teòrica: Informació i Fenomens Quàntics, Universitat Autònoma de Barcelona, ES-08193 Bellaterra (Barcelona), Spain; Department of Mathematics, University of Bristol, Bristol BS8 1TW, United Kingdom; and Centre for Quantum Technologies, National University of Singapore, Singapore

View Map $\theta$

(Received 19 January 2013; accepted 11 March 2013; published online 2 April 2013)

We establish a theory of quantum-to-classical rate distortion coding. In this setting, a sender Alice has many copies of a quantum information source. Her goal is to transmit a classical description of the source, obtained by performing a measurement on it, to a receiver Bob, up to some specified level of distortion. We derive a single-letter formula for the minimum rate of classical communication needed for this task. We also evaluate this rate in the case in which Bob has some quantum side information about the source. Our results imply that, in general, Alice's best strategy is a non-classical one, in which she performs a collective measurement on successive outputs of the source.

(C) 2013 American Institute of Physics

\section{Article Outline}

I. INTRODUCTION

II. NOTATIONS AND DEFINITIONS

III. DISTORTION OBSERVABLES

IV. QUANTUM-TO-CLASSICAL RATE-DISTORTION CODING

V. QUANTUM-TO-CLASSICAL RATE-DISTORTION CODING WITH QUANTUM SIDE INFORMATION

VI. CONCLUSIONS AND DISCUSSIONS
Alerts $\boldsymbol{P}$ Tools 9 Share

KEYWORDS and PACS

\section{Keywords}

encoding, quantum communication, quantum computing

\section{PACS}

03.67.Lx

Quantum computation architectures and implementations

$03.67 . \mathrm{Hk}$

Quantum communication

\section{ARTICLE DATA}

Digital Object Identifier

http://dx.doi.org/10.1063/1.4798396

\section{PUBLICATION DATA}

ISSN

0022-2488 (print)

1089-7658 (online)

Publisher

American Institute of Physics cross ref $_{\text {Member }}$

For access to fully linked references, you need to log in.

\section{References}

H. Barnum, "Quantum rate-distortion coding," Phys. Rev. A 62(4), 042309 (2000).

I. Devetak and A. Winter, "Classical data compression with quantum side information," Phys. Rev. A 68(4), 042301 (2003).

V. Giovannetti, S. Guha, S. Lloyd, L. Maccone, J. H. Shapiro, and H. P. Yuen, "Classical capacity of the lossy bosonic channel: The exact solution," Phys. Rev. Lett. 92(2), 027902 (2004)

B. Schumacher and M. A. Nielsen, "Quantum data processing and error correction," Phys. Rev. A 54, 2629-2635 (1996).

B. Schumacher and M. D. Westmoreland, "Sending classical information via noisy quantum channels," Phys. Rev. A 56(1), 131-138 (1997).

Figures (3)

Access to article objects (figures, tables, multimedia) requires a subscription; log in to view available files.

(Access to supplementary files, where available, is free for this journal.) 


\section{Related Articles (3)}

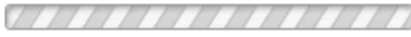

View More

\section{Your Recent History (3)}

\section{Recently Viewed}

- Quantum-to-classical rate distortion coding 